\title{
Role of 3D Volumetric and Perfusion Imaging for Detecting Early Changes in Pancreatic Adenocarcinoma
}

\begin{abstract}
Syed Rahmanuddin ${ }^{1 *}$, Ronald Korn ${ }^{2}$, Derek Cridebring ${ }^{3}$, Erkut Borazanci ${ }^{2}$, Jordyn Brase ${ }^{1}$, William Boswell ${ }^{1}$, Asma Jamil ${ }^{1}$, Wenli Cai ${ }^{4}$, Aqsa Sabir ${ }^{1}$, Pejman Motarjem ${ }^{1}$, Eugene Koay ${ }^{5}$, Anirban Mitra ${ }^{6}$, Ajay Goel ${ }^{7}$, Joyce $\mathrm{Ho}^{7}$, Vincent Chung ${ }^{7}$ and Daniel D. Von Hoff ${ }^{1,2,3}$

${ }^{1}$ National Medical Center \& Beckman Research Institute, City of Hope Comprehensive Cancer Center, Duarte, CA, United States, 2 Virginia G Piper Cancer Center, Honor Health, Scottsdale, AZ, United States, ${ }^{3}$ Molecular Medicine Division, Translational Genomics Research Institute (TGEN), Phoenix, AZ, United States, ${ }^{4}$ Department of Radiology, Massachusetts General Hospital, Boston, MA, United States, ${ }^{5}$ Department of Radiation Oncology, University of Texas MD Anderson Cancer Center, Houston, TX, United States, ${ }^{6}$ Department of Pathology, University of Texas MD Anderson Cancer Center, Houston, TX, United States, ${ }^{7}$ Molecular Diagnostic and Experimental Therapeutics, City of Hope Comprehensive Cancer Center, Monrovia, CA, United States
\end{abstract}

OPEN ACCESS

Edited by: Ilja Ciernik,

Städtische Klinikum Dessau, Germany

Reviewed by:

Sikandar Shaikh,

Shadan Hospital and Institute of Medical Sciences, India Jason Fleming,

Moffitt Cancer Center, United States

*Correspondence: Syed Rahmanuddin srahmanuddin@coh.org

Specialty section:

This article was submitted to Cancer Imaging and Image-directed Interventions, a section of the journal

Frontiers in Oncology

Received: 10 March 2021 Accepted: 13 August 2021 Published: 08 September 2021

Citation:

Rahmanuddin S, Korn R, Cridebring D,

Borazanci E, Brase J, Boswell W, Jamil A, Cai W, Sabir A, Motarjem P, Koay E, Mitra A, Goel A, Ho J, Chung $V$ and Von Hoff DD (2021) Role of $3 D$

Volumetric and Perfusion Imaging for Detecting Early Changes in

Pancreatic Adenocarcinoma.

Front. Oncol. 11:678617.

doi: 10.3389/fonc.2021.678617
Purpose: There is a major shortage of reliable early detection methods for pancreatic cancer in high-risk groups. The focus of this preliminary study was to use Time IntensityDensity Curve (TIDC) and Marley Equation analyses, in conjunction with 3D volumetric and perfusion imaging to demonstrate their potential as imaging biomarkers to assist in the early detection of Pancreatic Ductal Adenocarcinoma (PDAC).

Experimental Designs: A quantitative retrospective and prospective study was done by analyzing multi-phase Computed Tomography (CT) images of 28 patients undergoing treatment at different stages of pancreatic adenocarcinoma using advanced 3D imaging software to identify the perfusion and radio density of tumors.

Results: TIDC and the Marley Equation proved useful in quantifying tumor aggressiveness. Perfusion delays in the venous phase can be linked to Vascular Endothelial Growth Factor (VEGF)-related activity which represents the active part of the tumor. 3D volume analysis of the multiphase CT scan of the patient showed clear changes in arterial and venous perfusion indicating the aggressive state of the tumor.

Conclusion: TIDC and 3D volumetric analysis can play a significant role in defining the response of the tumor to treatment and identifying early-stage aggressiveness.

Keywords: 3D volumetric analysis, time intensity-density curve, perfusion analysis, pancreatic ductal adenocarcinoma, CT images

\section{INTRODUCTION}

Pancreatic cancer is one of the leading causes of cancer death globally (1). Due to conventional treatments having a minute impact on its natural progression, this disease is considered a major unresolved health problem. Metastases commonly develop in patients with pancreatic cancer resulting in high mortality (2). The average 5 -year survival rate is less than $5 \%$ and death can occur to most patients 6 months after cancer diagnosis $(1,3)$. The incidence of pancreatic cancer is higher 
in developed countries due, in part, to the differences in diet and lifestyle (1). Risk factors for pancreatic cancer include smoking cigarettes, a family history of pancreatic cancer, diabetes mellitus (Type I or Type II), obesity, consumption of a diet high in fat, alcohol use, and a lifestyle categorized by physical inactivity (4). One of the factors that contribute to the high rate of mortality stems from the nonspecific nature of early presenting symptoms. When the symptoms become severe enough for the individual to seek treatment, $80 \%-85 \%$ of patients present with the advanced unresectable form of this cancer (4). Although these symptoms are not specific and difficult to control, screening methods to identify the earlier stages of cancer can be addressed. Our primary focus for this preliminary study was to identify an approach that used the Time Intensity-Density Curve (TIDC) graphs to show its application in the early detection of pancreatic cancer. There are different types of pancreatic malignancies with Pancreatic Ductal Adenocarcinoma (PDAC) being the most common and severe, accounting for over $85 \%(4,5)$. The cure involves a complete removal of the tumor through surgery; however only $10 \%-20 \%$ of PDAC patients are surgically eligible at the time of diagnosis (4).To compound this problem further, there is a lack of effective early detection tools for pancreatic cancer in high-risk populations (those with greater than 5\% lifetime risk of pancreatic cancer) (4). Due to the lack of available early detection screening methods for pancreatic cancer, the fatality rates are greater, and the life expectancies from the time of diagnosis are lower as compared to other types of cancer $(5,6)$. Early screening methods for PDAC include Endoscopic Ultrasound (EUS) and Magnetic Resonance Imaging (MRI), which have not demonstrated improvement in the long-term survival (7) of patients. Despite the inability to enhance the long-term survival rates overall, these efforts in screening have paved the way for research in the creation of other modalities to detect pancreatic cancer. One of the current modalities is Computed Tomography (CT). CT exams are more accurate and robust in defining cancer characteristics while being the most common imaging modality available compared to EUS and MRI (7). Table 1 provides a list of current diagnostic modalities and their respective advantages and limitations.
The International Cancer of the Pancreas (CAPS) Consortium recommends individuals who fall into a high-risk group to get screened for cancer beginning at age 50 using EUS or MRI, with annual surveillance if no pancreatic lesions are identified on baseline assessment $(4,8)$. Both modalities are sensitive and specific for detecting small lesions or early cancer without the risks of radiation exposure. However, EUS performs better in cases of small solid lesions and provides an opportunity for tissue diagnosis by FNA, while MRI Cholangiopancreatography (MRCP) with and without contrast is preferred for cystic lesions and often used as a secondary form of screening in cases where there is a high clinical suspicion for pancreatic cancer despite a negative CT (7).

This study evaluated the use of $3 \mathrm{D}$ quantitative volumetric and TIDC analysis for detecting PDAC characteristics. We believed that the switch from $2 \mathrm{D}$ to $3 \mathrm{D}$ imaging would negate some of the limitations identified with 2D imaging. Multi-phase multi-detector computed tomography (MDCT) is the first line modality of choice for the diagnosis of pancreatic cancer (9). These tests must be tailored to the location of the area to be screened and use the available tools to complete the process, which can be difficult Pancreas-specific screening and diagnostic protocols involve the use of images in the pre-contrast, early arterial (CT angiography), pancreatic, and portal venous phases. The early arterial phase is useful in delineating the aorta and superior mesenteric artery and the portal venous phase can assess venous involvement and liver metastases. There is conflicting data from researchers regarding the optimal phase for enhancement of pancreatic lesions. Some studies suggest a better visualization of PDAC as low-attenuation lesions during the arterial phase due to its hypo-vascularity (4), whereas others demonstrated better pancreatic enhancement and attenuation differences between the normal pancreas and tumor in the portal venous phase (4).

Pancreatic tumors are often hypo-vascular and ill-defined, with irregular texture and abnormal morphology (10). To better understand the long-term outcomes and prognostic factors associated with PDAC, multi-phase CT images of patients undergoing treatment for PDAC were analyzed using advanced 3D imaging software to characterize the perfusion and radio density of tumors at various stages. Our goals were to identify

TABLE 1 | Benefits and limitations of pancreatic cancer diagnostic modalities i, ii

\begin{tabular}{|c|c|c|}
\hline Diagnostic modalities & Advantages & Limitations \\
\hline MDCT & Most commonly available & Nephrotoxicity \\
\hline $89 \%$ sensitivity & Best validated & Radiation exposure \\
\hline $90 \%$ specificity & Cheapest & \\
\hline MRI & Superior imaging & Expensive \\
\hline $89 \%$ sensitivity & Depicts local pancreatic disease & Less available \\
\hline $89 \%$ specificity & lodine-free and no radiation & Contraindicated with some metal implants \\
\hline EUS +/- FNA & Safe and less invasive & Less available in some countries \\
\hline $85 \%$ sensitivity ${ }^{\mathrm{iii}}$ & High sensitivity & Operator dependent \\
\hline \multirow[t]{2}{*}{$96 \%$ specificity ${ }^{\mathrm{iii}}$} & Able to detect small lesions & Inability to detect distant metastasis \\
\hline & Able to take histological sample & \\
\hline
\end{tabular}

'Adapted from Zhang L et al., World Journal of Gastroenterology. 2018; 24:2047-2060. ${ }^{6}$

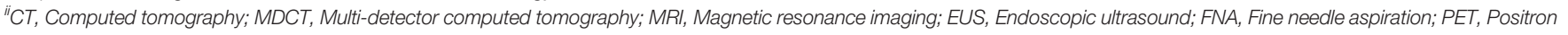
emission tomography.

${ }^{\text {iii }}$ Pooled sensitivity and specificity for EUS with FNA. 
imaging biomarkers to help detect pancreatic cancer early and define features of pancreatic cancer that correlate with disease severity and treatment response. The findings from this study might help define the mechanisms and growth patterns of PDAC while laying the groundwork for further research in early PDAC detection.

\section{MATERIALS AND METHODS}

\section{Patient Population}

A quantitative, retrospective, and prospective study was done using 41 adult patients with unresectable treatment naïve PDAC who were either enrolled in a clinical trial at Honor Health Cancer Center (Phoenix, AZ) or treated at City of Hope Comprehensive Cancer Center (Duarte, CA) as Standard of Care (SOC) non-study patients between 2015 and 2019.Consent was obtained from all clinical trial patients for the use of their images gathered from medical records for imaging analysis. As a result, there was limited control over the way the images were taken or its time frames. All patients were over the age of 18 and had been diagnosed with PDAC. We divided the participant sample into two groups, labeled Cohort 1 and Cohort 2.

Cohort 1 consisted of 22 patients enrolled in the clinical trial treated at Honor Health Cancer Center. Patients within this sample group received neo-adjuvant therapy (paricalcitol, paclitaxel protein bound, cisplatin, and gemcitabine) for up to 6 months with standard of care CT multiphase imaging. The original purpose for the Honor Health clinical trial was to see the resectability and CA 19-9 status within non-metastatic pancreatic cancer but we decided to use the preliminary imaging findings in this ongoing study and assess the volumetric response in Cohort 1 as well as Cohort 2. Patients with a positive metastatic disease were excluded from the study. Furthermore, patients whose tumors were deemed resectable after the completion of neo-adjuvant therapy were likewise removed from the study to receive SOC treatment due to changes in their condition.

Cohort 2 consisted of 19 patients treated at the City of Hope Comprehensive Cancer Center. Patients within this sample group received SOC therapy only and the CT multiphase imaging exam was performed at least every 3 months as a follow-up scan. Like Cohort 1, neo-adjuvant rules were not applied since most images were obtained retrospectively. Cohort 2 was divided based on the location where participants received care to ensure that variations in treatment, tools, or other variables did not affect the participants within their given groups. To make sure that there was uniformity in the participant samples (i.e., each patient in a participant group was exposed to similar variables as the other patients within the same participant group), we worked to increase the overall reliability of the data collected during the image acquisition process.

We submitted the complete requests for information in conjunction with the Health Insurance Portability and Accountability Act (HIPPA) authorization to obtain the records of all participants from the Honor Health and City of Hope Medical Records Departments. Following the receipt of the images, we then analyzed the scans to ensure that the images fit the necessary criteria. However, since we had no control over the image collection process, most of the data obtained could not be used due to issues with the imaging protocol. Additional exclusion criteria for these images included poor image quality, variations in time between images (e.g., too far apart or noncomparable to others within the sample set), and the use of nonstandard practices in image collection. These variations, especially the latter, occurred more frequently than we anticipated, leaving the image sample size for TIDC at 28 patients out of the anticipated 41 participants from which scans were requested.

\section{Image Acquisition}

For the PDAC cohorts, we required multiphase CT images for collection at Honor Health and City of Hope. All images from Honor Health and City of Hope used the same standard protocol to acquire the participant CT scans for analysis. First, the images were obtained from the imaging conducted with multi-phase CT. Most of the acquired images were scanned on a GE scanner with a standard pancreatic protocol which included pre-injection, arterial, and portal venous phases (11). Second, to maximize the coherency and accuracy of the analysis, the images must have used the standard iodinated contrast agent Isovue 370. Patient files from both cohorts indicated that each participant received a dose of $150 \mathrm{cc}+40 \mathrm{cc}$ of saline that was injected at a rate of $5 \mathrm{cc}$ per second into the antecubital vein of the participant.

For scanning parameters, the areas of interest were defined as the region above the diaphragm to the inferior liver margin for arterial phase images. The region from above the diaphragm to the superior aspect of the iliac crest was used for both precontrast and venous phase images. Multi-phase scanning parameter used in the image collection process was at $120 \mathrm{kV}$ with a 0.5 second rotation time. All images were reconstructed as follows: pre-contrast phase images were reconstructed into 3$\mathrm{mm}$ slices and images from arterial or venous phases into $2-\mathrm{mm}$ slices. Images were reviewed by experienced radiologists to ensure that the image quality was sufficient but 3D Qi has some high-resolution issue which can be fixed in future upgrades. Of the 28 participant images obtained, 24 were of ample quality for analysis. Initial images were reconstructed into $0.625 \times 1.25 \mathrm{~mm}$-slices for 3D post-processing and uploaded from the Picture Archiving and Communication System (PACS) to advanced imaging software (GE Advantage Workstation 3.2) for volumetric analysis.

\section{D Volumetric Analysis}

A 3D imaging software application called GE AW 3.2 USA was used on the 28 PDAC patient CT images to conduct the volumetric analyses. This method of analysis refers to the total amount of a given substance, in this case, the pancreatic tumor, which was determined by measuring the volume that the tumor occupied (12-14). Similar analytical approaches had promising outcomes when exploring various medical conditions involving the pancreas and liver, providing adequate justification for its use 
in the context of this study (12-17). To confirm the accuracy of this method, multiple series were registered and regions of interest were identified prior to receiving the requested study images to define volumetric tumor quantification. Edits to the regions of interest were performed when needed to ensure that volumetric analysis was as accurate as possible using the available tools (see Figure 1).

Images were imported to the Massachusetts General Hospital application called 3D Quantitative Imaging software (3DQI). The PDAC tumor was identified by a radiologist and radiology expert. All series were opened side by side to do a comparison for better visibility and quantitative assessment (See Figure 2). Elaboration on the perfusion imaging characteristics on multiphase CT was a major goal of this research. The standard CT protocol for the PDAC imaging requires noncontrast with arterial and venous phase imaging. This preliminary study was designed to observe changes occurring during the wash-in and wash-out rates seen in perfusion imaging. This application has been used successfully in hepatocellular carcinoma (HCC) (18-21). HCC shows a complete wash-out in the venous and late phase imaging but the PDAC tumor becomes more vascularized in the venous phase which indicates missing information that might be critical to how PDAC is defined.

\section{Marley Equation}

The Marley equation was created and first investigated in the Rahmanuddin lab to describe the difference in the enhancement patterns between the arterial and venous phases (see Figure 3). This equation uniquely defines the characteristics of each pixel and flow inside the tumor which is aggressive in nature and can potentially be useable in the early detection of pancreatic cancer.
In comparison to $\mathrm{HCC}$ imaging, PDAC has unique characteristics which are seen in the late arterial or venous phase imaging. It is visible in the venous phase which indicates the prolonged time of tumor vascularization. The longer the venous phase wash-out rate, the higher the tumor aggression. Marley equation helps to define the gap between the arterial and venous phases which can estimate the accurate change in the time on perfusion imaging. Our previous preliminary results showed that using the Marley Equation can be beneficial in observing the differences more precisely. This equation might be helpful in relating imaging perfusion to Vascular Endothelial Growth Factors (VEGF). Figure 3 provides a breakdown of the Marley equation, indicating how it was used in the assessment within the current study.

\section{Time-Intensity-Density Curves}

The potential in the application of TIDC, as a means of detecting tumor aggressiveness was seen using 3DQI software. TimeDensity Curves for CT scans and Time-Intensity Curves for MRI scans provide a visual tool for the enhancement or reduction of a particular mass/lesion over time based on the intensity and density (22-25). Previous studies have demonstrated the utility of TIDC analysis in differentiating solid lesions in the pancreas using contrast-enhanced EUS (16), a first line diagnostic test (26-29). However, due to the limitations presented in the US examinations, compared to the test environment setting, and the associated concerns regarding accuracy across the application throughout treatment facilities in the United States, past researchers have noted the difficulties associated with standardizing these markers as a means of creating a unified evidence-based practice (EBP) for its use as a diagnostic modality (30-34).
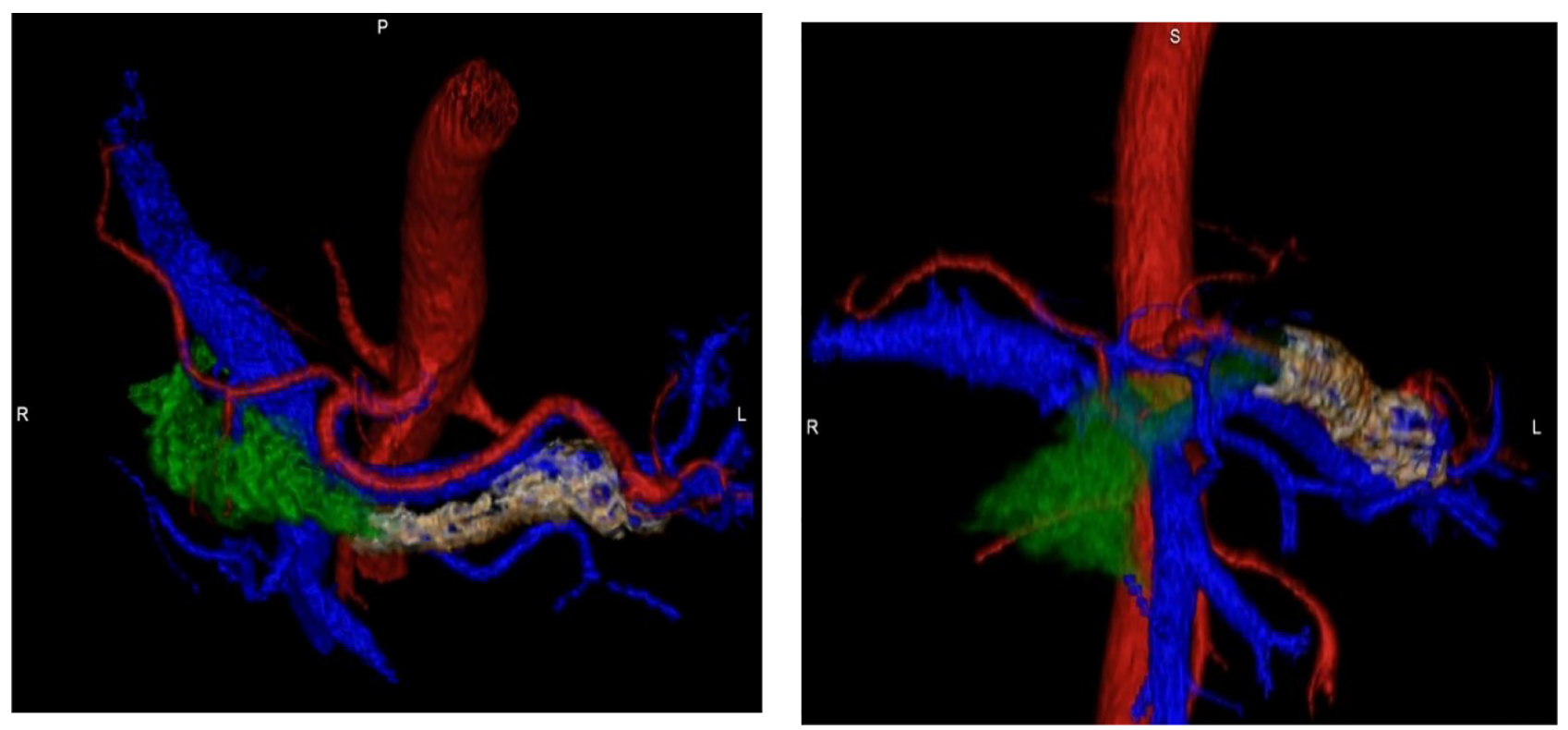

FIGURE 1 | Pancreatic Tumor Volumetric Mapping. 


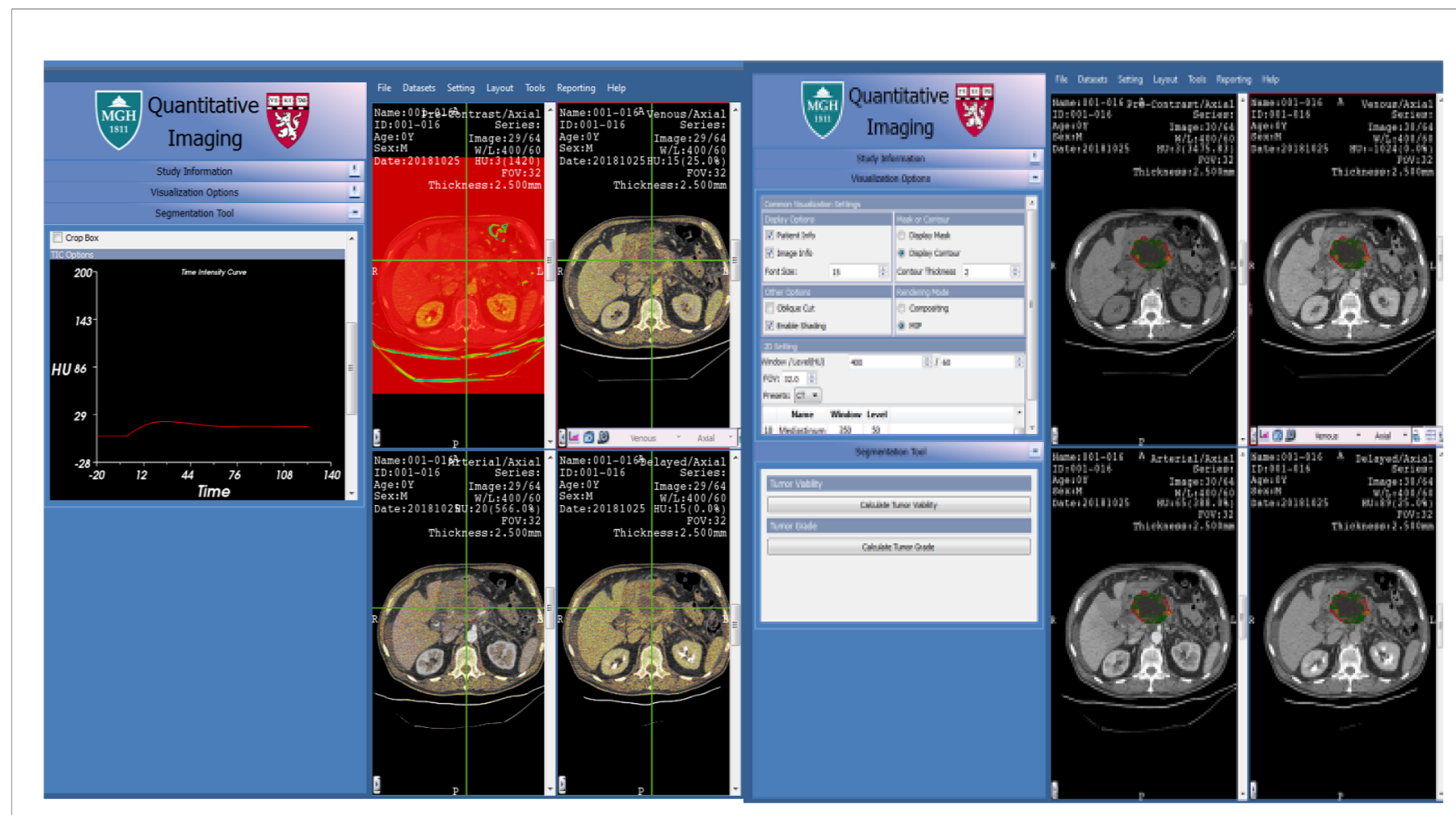

FIGURE 2 | 3D Qi Software.

Our focus was to identify an approach that used the TIDC to show how it can be applied in the early detection of pancreatic cancer. The identification of the TIDC patterns that correlate with PDAC responses to treatment would enable clinicians to better evaluate the severity of the disease, in addition to serving as a diagnostic screening modality. The TIDC model generated for the current study displayed information based on each pixel present in the multi-phase CT imaging within the tumors and tissues of interest in each participant. Through the integration of a computer-based software for pixel-by-pixel analysis, we were able to define the time-in and time-out quantitative numbers with a greater level of precision compared to past efforts (35-38). The artificial intelligence tool used was uniquely designed to differentiate the pixel-based change in contrast enhancement related to perfusion in multiphase imaging.

\section{RESULTS}

The Marley equation has proven that it is applicable in quantifying tumor aggressiveness. Using this equation, we were able to generate normal distribution histograms based on the minimum, maximum, and average tumor radio density, as measured in Hounsfield Units (HU). Tissue radio density refers to the opacity of the tissue as measured by how readily the different types of electromagnetic radiation passes through

\section{Marley Equation:}

$$
\text { Area under the normal distributions }=\left|\int_{x_{\text {venous }} \text { min }}^{x_{\text {arterial }} \text { max }}\left[f\left(x_{\text {venous }}\right)-f\left(x_{\text {arterial }}\right)\right] d x\right|
$$

$\mathrm{X}$ is defined as the range of perfusion values in the arterial or venous phase.

$$
\text { The function } \mathrm{f}(\mathrm{x}) \text { represents } f(x)=\frac{1}{\sigma \sqrt{2 \pi}} e^{-\frac{(x-\mu)^{2}}{2 \sigma^{2}}}(\sigma \text { is variation } \& \pi \text { is the average })
$$




\section{Normal}

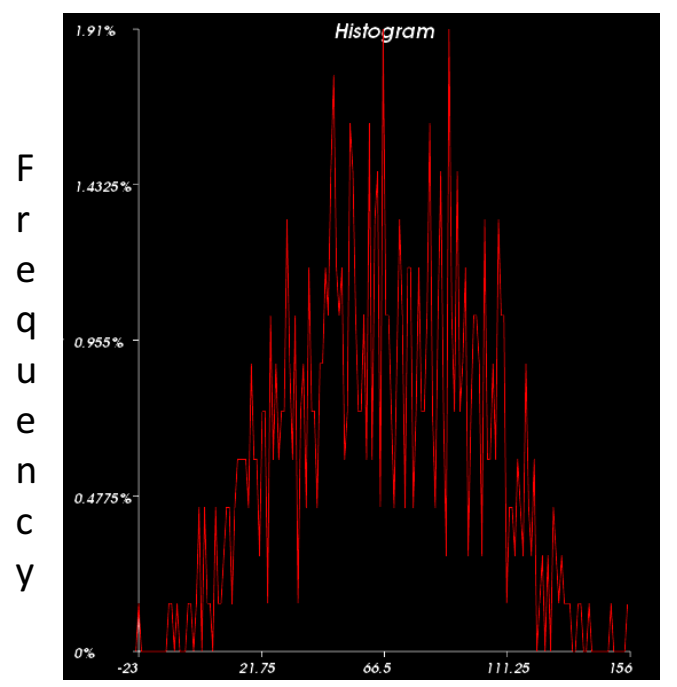

$\mathrm{HU}$
Tumor

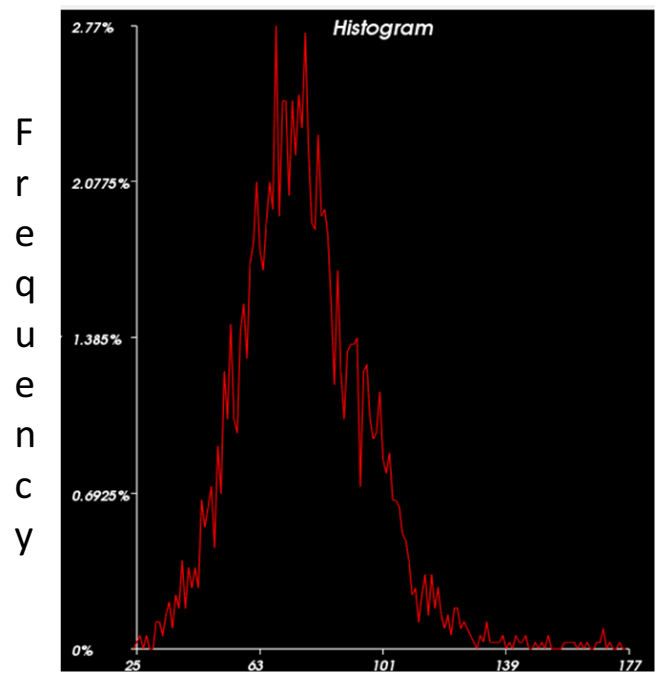

$\mathrm{HU}$

FIGURE 4 | Histogram Analysis of Normal Pancreas vs. PDAC. Histogram distribution of the normal pancreas (left) shows greater variation in density representative of its heterogeneous echotexture compared to a more homogenous pattern in PDAC (right).

the tissue itself. HU is a common scale used in assessing CT scans and refers to a linear transformation of the radio density of various materials. The common HU reference point is distilled water at a standard pressure and room temperature set as zero. The tissue radio density on CT images, measured in $\mathrm{HU}$, changed with respect to time. Specifically, analysis showed that the difference in tumor perfusion during the arterial and venous phases increases with respect to time. This change continuously increases during the later stages of the venous phase without changing the tumor dynamic contrast enhancement.

Our preliminary study highlighted the following major areas based on perfusion imaging. The Marley equation showed that perfusion changes play a major role in tumor kinetics (see Figure 4). PDAC tumor morphology is unique with normal heterogeneous features which have characteristics opposite to that of liver tumor morphology $(4,6,19,21)$. This is an important result due to the application of this equation showing a distinct contrast between the healthy pancreas and tumor.

TDIC analysis was performed on all patient images due to the limited availability of the multiphase standard imaging protocol. This was caused by missing scans including non-contrast images or series of the arterial or venous phases. However, $3 \mathrm{D}$ volumetric quantification was conducted on all images. Based on the pilot data, there are indications that perfusion in PDAC exhibits delays, showing a later response, as compared to a normal pancreatic tissue (see Figure 5). TIDC analysis shows that perfusion takes a longer time in the venous phase in PDAC imaging. These perfusion delays represent the active form of the tumor which might be significantly associated with tumor aggressiveness or VEGF-related activity. Longer perfusion time in the venous phase was directly correlated with the aggressiveness of the disease. This groundbreaking finding shows that TIDC analyses can observe PDAC in the venous phase manifesting in a manner opposite the normal pancreatic morphological TIDC which cleared the dynamic contrast in the late venous phase.

\section{D Volumetric Analysis}

3D tumor volume was measured in patients diagnosed with PDAC receiving standard treatment with or without neoadjuvant therapy. A correlation of disease burden decreasing with clinical treatment was seen predominantly in Cohort 1 . Changes in arterial and venous perfusion were related to tumor aggression. These findings have the potential for integration to create new screening tools which may serve as potential imaging biomarkers for the early detection of PDAC. Due to the small sample size, we were unable to produce statistical analyses, but the hypothesis is completely based on the perfusion characteristics on the follow-up imaging. Based on the findings, the PDAC volume measure decreases constantly over time which is seen with eight of the patients in Cohort 1 (see Figure 6), which indicates that the neoadjuvant population had a better control on the disease compared to the SOC of Cohort 2. The graphs indicated that $3 \mathrm{D}$ volume measurements might be a potential imaging biomarker to identify the disease progression or regression status. $3 \mathrm{D}$ volumetrics can provide more precise pixels based on the quantitative assessment on follow-up imaging for the those previously diagnosed with PDAC.

The average percent change in volume based on the images analyzed was explored (see Figure 7). Serial imaging in Cohort 1, who were receiving neoadjuvant therapy in addition to SOC 

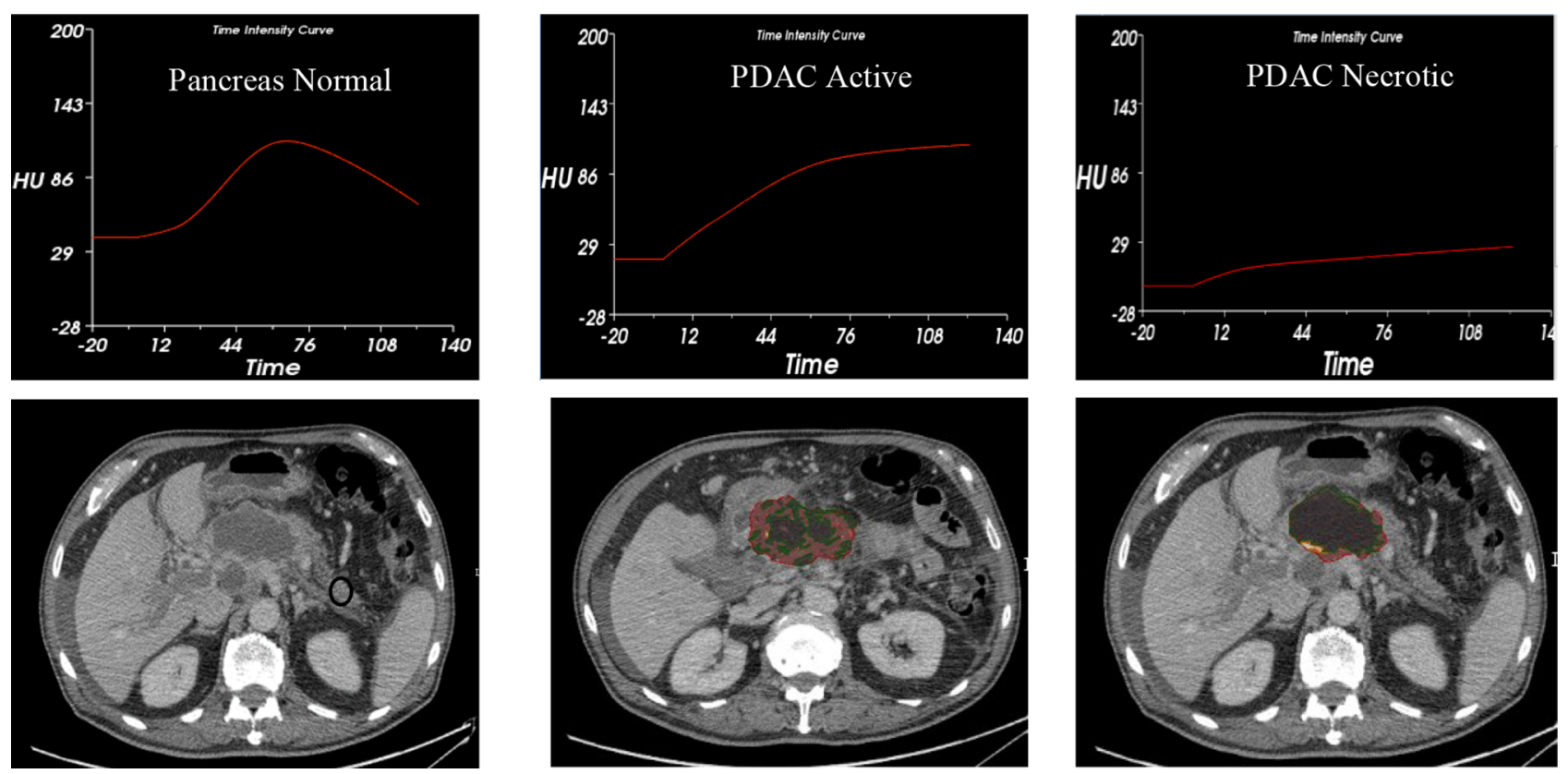

FIGURE 5 | Time Intensity/Density Curves.

treatment, showed steady decreases in tumor volume and venous vasculature. Imaging from Cohort 2, who were receiving standard therapy alone, displayed variable responses during treatment with an average decrease overall. This change does not display major differences compared to Cohort 1 but shows some change on the follow-up imaging. $3 \mathrm{D}$ volume could be a main indicator for the major and minor volumetric change compared to that of $2 \mathrm{D}$ analysis.

\section{DISCUSSION}

Pancreatic ductal adenocarcinoma (PDAC) accounts for over $85 \%$ of pancreatic malignancies (1). Despite the recent improvements to diagnostic imaging, conventional treatments, and newer targeted therapies, mortality remains high (4). The deep retroperitoneal location of the pancreas creates certain diagnostic uncertainties that limit current efforts in the early detection of pancreatic cancer (4). Multiphase CT is currently the standard modality for diagnostic testing (39-42). However, changes to tissue morphology that occur with PDAC make it difficult to define texture accurately with 2D CT imaging (39). This study evaluated the use of $3 \mathrm{D}$ quantitative volumetric and TIDC analysis for detecting PDAC treatment response.

The difference in tumor perfusion during the arterial and venous phases increased with respect to time. Results showed that volume- and perfusion-based imaging biomarkers could play a significant role in defining the aggressiveness of early-stage

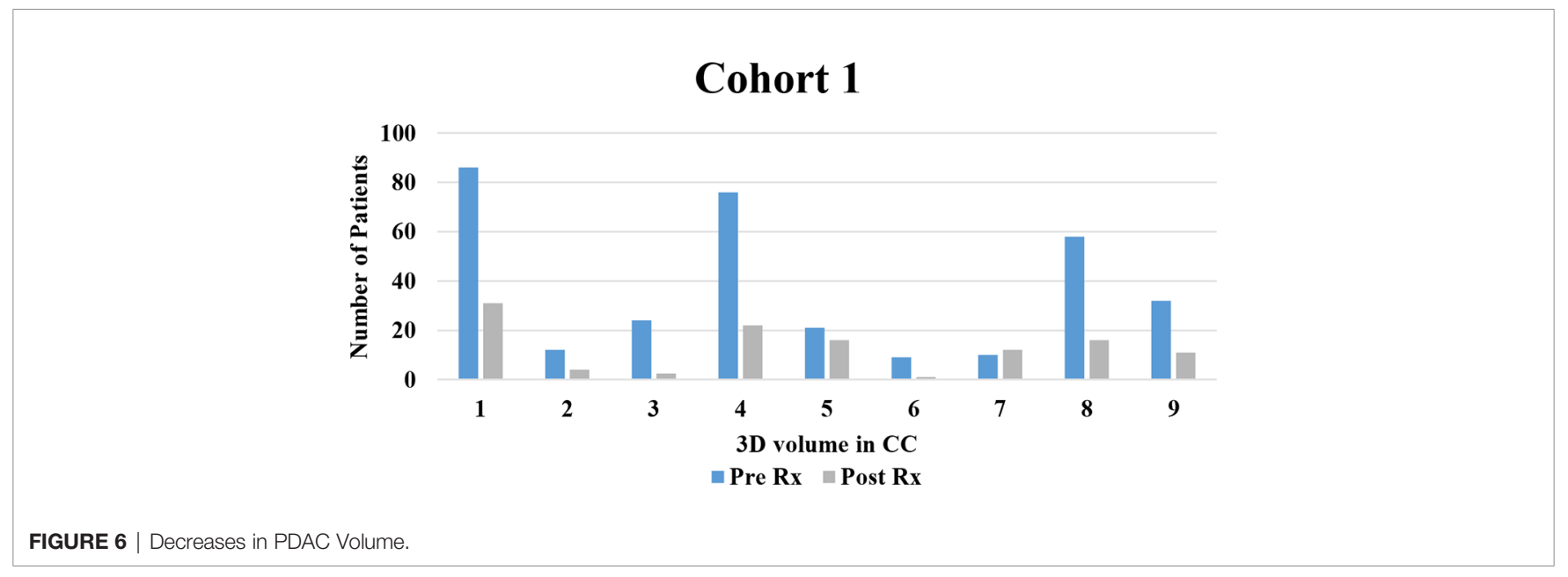




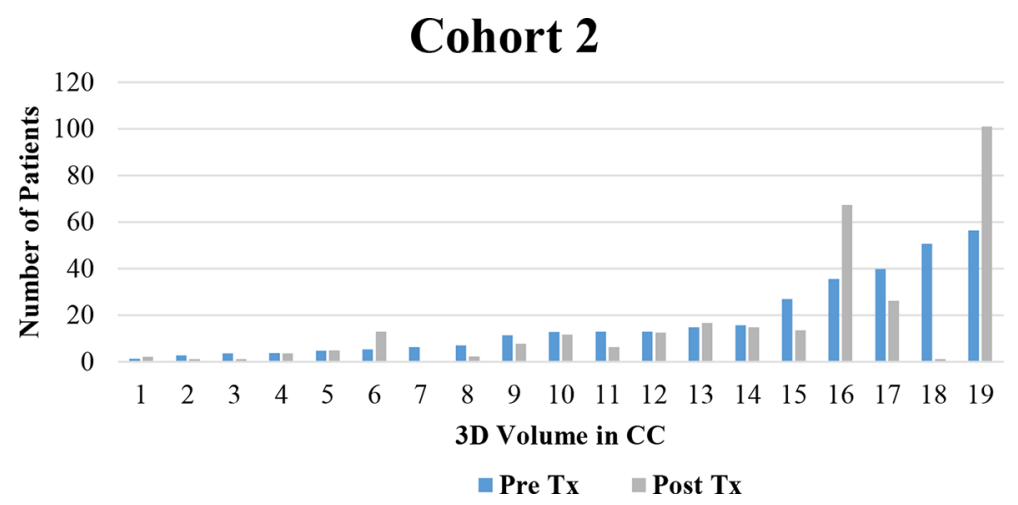

FIGURE 7 | Change in 3D volume which decreased over time.

tumors and response to therapy. Further studies to identify additional 3D imaging biomarkers for pancreatic cancer could collectively increase the precision of early diagnostic imaging (See Figure 8). Based on the results, it is predicted that the longer the time spent in the venous phase might relate to the tumor aggressiveness behavior. This methodology in conjunction with the Marly Equation might be useful for the early detection of pancreatic cancer by quantifying the exact changes in the perfusion imaging. The larger the change, the more aggressive the disease. It can also be useful for comparing TIDC standard venous phase perfusion rates to PDAC cases.

Tissue radiodensity on CT images, measured in HU, changed with respect to time. Clear documentation of these changes, albeit in a small sample size, suggests that there is potential for application in the creation of a screening tool that might be used to detect PDAC; however, more information is needed. Conclusive evidence that finds this result as accurate across a larger and more diverse sample population might point toward the use of this application in diagnostic modalities. Perfusion delays represented the active form of the tumor which might be associated with
VEGF-related activity. Longer perfusion time in the venous phase was directly correlated with the aggressiveness of the disease which might reflect an increased angiogenesis. In cancer, VEGF has been identified as one of the main culprits behind rapid angiogenesis which deprives healthy cells of sufficient nutrients and promotes metastases (43). Even though PDAC displays a poor perfusion, it might still have a considerable VEGF-related activity (44). Many tumors require angiogenic processes that are therapeutically targetable (45). However, this dependency indicates the demand for nutrients and oxygen exchange which is satisfied by the metabolic burden of tumor cells instead of a measure of angiogenic dependence (45). Future projects with larger patient populations might provide more insight into the relationship of PDAC aggressiveness and angiogenesis.

This was a retrospective study, which means that contact from two medical facilities from which the participant data were obtained was not standardized. As a result, several potential participants were excluded from this study due to variability in the acquisition times of images during the treatment regimens. Patients at one facility receiving a certain type of treatment may
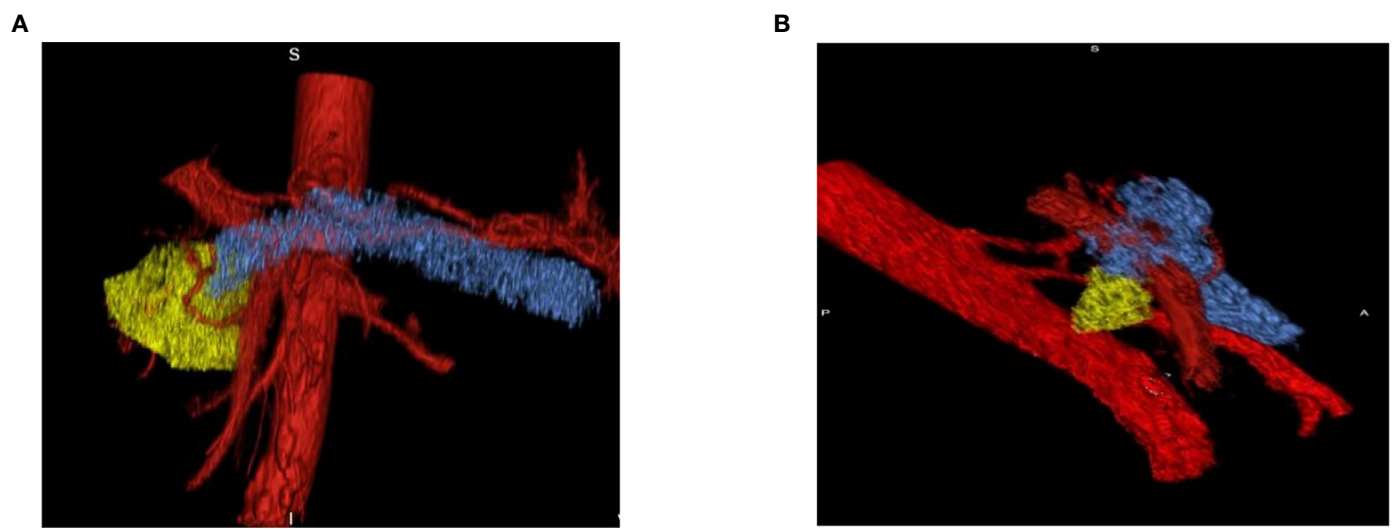

FIGURE 8 | Pre- and Post-Treatment Tumor. (A) Pre-treatment tumor surrounded by vessels which indicates the increase in perfusion effect in tumor progression. (B) Post-treatment tumor volume decreases on the follow-up scan which shows that decreases in perfusion lessens the aggressiveness of the tumor. 
have images taken within a particular time frame after receiving treatment, while others may have images taken within a different time frame. This may cause imaging inconsistencies across the patient data that might not have been clearly documented, making it difficult to identify confounding variables and analysis less effective.

\section{CONCLUSION}

The clinical application of TIDC analysis is an emerging area of perfusion imaging. Previous studies have demonstrated the utility of TIDC analysis in differentiating pancreatic solid lesions using contrast-enhanced endoscopic ultrasound. However, technician-dependent ultrasound-based techniques have been shown, historically, to be difficult to standardize and can have limited accuracy. Variations in the way technicians complete their assigned imaging tasks can result in slightly different images. Similarly, differences in available imaging technologies within treatment facilities contribute to further variations in standardization. However, increased pushes for the integration of EBP within medical screening and diagnostic modalities could suggest that future efforts in this area could be better received, with a greater potential for application. Creating a unified standard set of protocols and procedures to assess multiphase CT scans is needed, regardless of instrumentation. Focus on the types of content to be included in the images, and the different views of the area should be increased in current screening methods. This information can then be translated into different diagnostic methods that can improve the effectiveness of early detection practices; however, the integration of EBP is only the first step.

TIDC analysis in six patients demonstrated a late venous perfusion delay, as compared to perfusion rates for a normal pancreas. It was hypothesized that the perfusion patterns in the late venous phase could be used to define the aggressiveness of pancreatic cancer. Our research was limited by the small sample size of the study. It was anticipated to collect and analyze data from all patient files; however, there was only enough uniformity in the imaging practices and time frames of six participants. This limitation is brought by a lack of imaging protocols with sufficient acquisition times suggesting an area in need for improvement. Several of these concerns can be decreased and possibly mitigated entirely with the integration of clear EBPs. The construction of TIDCs requires the availability of images taken from multiple phases over a longer period of time than that which is utilized most often in standard pancreatic imaging

\section{REFERENCES}

1. Veisani Y, Jenabi E, Khazaei S, Nematollahi S. Global Incidence and Mortality Rates in Pancreatic Cancer and the Association With the Human Development Index: Decomposition Approach. Public Health (2018) 156:87-91. doi: 10.1016/j.puhe.2017.12.015

2. Li D, Xie K, Wolff R, Abbruzzese JL. Pancreatic Cancer. Lancet (2004) 363 (9414):1049-57. doi: 10.1016/S0140-6736(04)15841-8 protocols. Among the study images obtained, the peak or maximum value of the curve was not visualized for pancreatic cancer images which suggests the need for extended acquisition times. It was suggested that the time-to-peak on TIDCs might provide important information about tumor aggressiveness. It might be helpful to define the precision-based imaging findings for the detection of the PDAC. Mostly patients who are diagnosed with cancer go the SOC protocol which provides the EBP clinical information.

Our preliminary results can be beneficial in acquiring more precise information for the tumor behavior using the perfusion imaging. Projects are currently underway that optimize imaging protocols for TIDC analysis in pancreatic cancer. With this study using a smaller population, we plan to take these new imaging techniques to a larger and more diverse population to test TIDC. Through the proliferation of research in this area, the creation of an early screening tool for pancreatic cancer becomes even more likely. Rahmanuddin lab is currently working on the discovery of several imaging biomarkers based on the perfusion imaging for PDAC.

Pancreatic cancer continues to have a high mortality due to the late detection and lack of effective screening methods. The novel radiomic biomarkers described here can be applied towards detecting variations in pancreatic cancer. A potential future screening tool for pancreatic cancer can involve a combination of standard imaging protocols, quantitative imaging biomarkers, and targeted molecular assays that allow the earlier detection of pancreatic cancer in high-risk patient populations. The completion of this study, combined with the efforts of other researchers in this and similar areas of research, serves as the first step in the creation of a screening tool of this nature. Using a smaller sample size, the potential effectiveness of TIDC in applications for screening was demonstrated.

\section{DATA AVAILABILITY STATEMENT}

The raw data supporting the conclusions of this article will be made available by the authors, without undue reservation.

\section{AUTHOR CONTRIBUTIONS}

All authors were involved in study design, manuscript writing, collection, and the final submission process. All authors contributed to the article and approved the submitted version.
3. Ben Q, Xu M, Ning X, Liu J, Hong S, Huang W, et al. Diabetes Mellitus and Risk of Pancreatic Cancer: A Meta-Analysis of Cohort Studies. Eur J Cancer (2011) 47(13):1928-37. doi: 10.1016/j.ejca.2011.03.003

4. Vincent A, Herman J, Schulick R, Hruban R, Goggins M. Pancreatic Cancer. Lancet (2011) 378(9791):607-20. doi: 10.1016/S0140-6736(10) 62307-0

5. Ilic M, Ilic I. Epidemiology of Pancreatic Cancer. World JWenal Gastroenterol (2016) 22(44):9694-705. doi: 10.3748/wjg.v22.i44.9694 
6. Zhang Q, Zeng L, Chen Y, Lian G, Qian C, Chen S, et al. Pancreatic Cancer Epidemiology, Detection, and Management. Gastroenterol Res Pract (2016) 2016:1-10. doi: 10.1155/2016/8962321

7. Zhang L, Sanagapalli S, Stoita A. Challenges in Diagnosis of Pancreatic Cancer. World JWenal Gastroenterol (2018) 24(19):2047-60. doi: 10.3748/ wjg.v24.i19.2047

8. Canto M, Harinck F, Hruban R, Offerhaus G, Poley J, Jamel I, et al. International Cancer of the Pancreas Screening (CAPS) Consortium Summit on the Management of Patients With Increased Risk for Familial Pancreatic Cancer. Gut (2013) 62(3):339-47. doi: 10.1136/gutjnl-2012303108

9. Choi B, Chung M, Han J, Han M, Yoon Y. Detection of Pancreatic Adenocarcinoma: Relative Value of Arterial and Late Phases of Spiral CT. Abdominal Imaging (1997) 22(2):199-203. doi: 10.1007/s002619900172

10. Crippa S, Partelli S, Bassi C, Beradi R, Capelli P, Scarpa A, et al. Long-Term Outcomes and Prognostic Factors in Neuroendocrine Carcinomas of the Pancreas: Morphology Matters. Surgery (2016) 159(3):862-71. doi: 10.1016/ j.surg.2015.09.012

11. Graf O, Boland G, Warshaw A, Fernandez-del-Castillo C, Hahn P, Mueller P. Arterial Versus Portal Venous Helical CT for Revealing Pancreatic Adenocarcinoma: Conspicuity of Tumor and Critical Vascular Anatomy. Am J Roentgenol (1997) 169(1):119-23. doi: 10. 2214/ajr.169.1.9207510

12. Phillip V, Zahel T, Danninger A, Erkan M, Dobritz M, Steiner J, et al. Volumetric Gain of the Human Pancreas After Left Partial Pancreatic Resection: A CT-Scan Based Retrospective Study. Pancreatology (2015) 15 (5):542-7. doi: 10.1016/j.pan.2015.06.007

13. Baliyan V, Kordbacheh H, Parakh A, Kambadakone A. Response Assessment in Pancreatic Ductal Adenocarcinoma: Role of Imaging. Abdominal Radiol (2018) 43(2):435-44. doi: 10.1007/s00261-017-1434-7

14. Maignan A, Ouaissi M, Turrini O, Regenet N, Loundou A, Louis G, et al. Risk Factors of Exocrine and Endocrine Pancreatic Insufficiency After Pancreatic Resection: A Multi-Center Prospective Study. JWenal Visceral Surg (2018) 155 (3):173-81. doi: 10.1016/j.jviscsurg.2017.10.007

15. Pandharipande P, Krinsky G, Rusinek H, Lee V. Perfusion Imaging of the Liver: Current Challenges and Future Goals. Radiology (2005) 234:661-73. doi: 10.1148/radiol.2343031362

16. Demir I, Jager C, Friess H, Ceyhan G. The Impact of Resection Margin Status on Pancreatic Cancer Associated Survival: A Meta-Analysis. HPB (2016) 18 (supplement 1):e-130-131. doi: 10.1016/j.hpb.2016.02.307

17. Reichman T, Fiorello B, Carmody I, Bohorquez H, Cohen A, Seal J, et al. Using on-Site Liver 3-D Reconstruction and Volumetric Calculations in Split Liver Transplantation. Hepatobiliary Pancreatic Dis Int (2016) 15(6):587-92. doi: 10.1016/S1499-3872(16)60155-8

18. Nayak A, Kayal E, Arya M, Culli J, Krishan S, Agarwal S, et al. ComputerAided Diagnosis of Cirrhosis and Hepatocellular Carcinoma Using MultiPhase Abdomen CT. Int J Comput Assist Radiol Surg (2019) 14(8):1341-52. doi: 10.1007/s11548-019-01991-5

19. Roberts L, Sirlin C, Zaiem F, Almasri J, Prokop L, Heimbach J, et al. Imaging for the Diagnosis of Hepatocellular Carcinoma: A Systematic Review and Meta-Analysis. Hepatology (2018) 67(1):401-21. doi: 10.1002/hep.29487

20. Pickhardt P, Kitchin D, Lubner M, Ganeshan D, Bhalla S, Covey A. Primary Hepatic Angiosarcoma: Multi-Institutional Comprehensive Cancer Centre Review of Multiphasic CT and MR Imaging in 35 Patients. Eur Radiol (2015) 25(2):315-22. doi: 10.1007/s00330-014-3442-0

21. Lo E, Rucker A, Federle M. Hepatocellular Carcinoma and Intrahepatic Cholangiocarcinoma: Imaging for Diagnosis, Tumor Response to Treatment and Liver Response to Radiation. Semin Radiat Oncol (2018) 28(4):267-76. doi: 10.1016/j.semradonc.2018.06.010

22. Radiant Viewer. Time-Intensity Curves (TIC) (2016). Available at: https://www. radiantviewer.com/dicom-viewer-manual/time-intensity_curves_tic.htm.

23. Krix M. Time Intensity Curves. In: Baert A.L. (eds). Encyclopedia Diagn Imaging. Berlin, Heidelberg: Springer (2008). doi: 10.1007/978-3-540-352808_2488

24. Fellner C, Prantl L, Rennert J, Stroszczynski C, Jung E. Comparison of TimeIntensity Curve- (TIC-) Analysis of Contrast-Enhanced Ultrasounce (CEUS) and Dynamic Contrast-Enhanced (DCE) MRI for Postoperative Control of
Microcirculation in Free Flaps - First Results and Critical Comments. Clin Hemorheology Microcirculation (2011) 48(1):187-98. doi: 10.3233/CH-2011-1399 25. Sherif H, Mahfouz A, Kambal A, Sayedin A, Mujeeb I. Relative Time-Intensity Curve: A New Method to Differentiate Benign and Malignant Lesions on Breast MRI. Cancer Imaging (2014) 14(supplement 1):26. doi: 10.1186/14707330-14-S1-P26

26. Yamamoto N, Kato H, Tomoda T, Matsumoto K, Sakakihara I, Noma Y, et al. Contrast-Enhanced Harmonic Endoscopic Ultrasonography With TimeIntensity Curve Analysis for Intraductal Papillary Mucinous Neoplasms of the Pancreas. Endoscopy (2016) 48(1):26-34. doi: 10.1055/s-0034-1393563

27. Choi T, Lee J, Kim J, Yu M, Han J, Choi B. Comparison of Multidetector CT and Gadobutrol-Enhanced MR Imaging for Evaluation of Small, Solid Pancreatic Lesions. Korean J Radiol (2016) 17(4):509-21. doi: 10.3348/ kjr.2016.17.4.509

28. Granata V, Fusco R, Catalano O, Setola S, Castelguidone E, Piccirillo M, et al. Multidetector Computer Tomography in the Pancreatic Adenocarcinoma Assessment: An Update. Infect Agents Cancer (2016) 11:57. doi: 10.1186/ s13027-016-0105-6

29. Garcia-Vazquez V, Marinetto E, Guerra P, Valdiviesco-Casique M, Calvo F, Alvarado-Vasquez E, et al. Assessment of Intraoperative 3D Imaging Alternatives for IOERT Dose Estimation. Seitschrift fur Medizinishce Physik (2017) 27(3):218-31. doi: 10.1016/j.zemedi.2016.07.002

30. Pomara C, Bello S, Grilli G, Guglielmi G, Turillazzi E. Multi-Phase Postmortem CT Angiography (MPMCTA): A New Axillary Approach Suitable in Fatal Thromboembolism. La Radiologica Med (2015) 120 (7):670-3. doi: 10.1007/s11547-014-0467-z

31. Gupta V, Lantz J, Henriksson L, Engvall J, Karlsson M, Persson A, et al. Automated Three-Dimensional Tracking of the Left Ventricular Myocardium in Time-Resolved and Dose-Modulated Cardiac CT Images Using Deformable Image Registration. JWenal Cardiovasc Computed Tomography (2018) 12(2):139-48. doi: 10.1016/j.jcct.2018.01.005

32. Mokrane F, Dercle L, Myrignac O, Crubezy E, Rousseau H, Telmon N, et al. Towards a Multi-Phase Postmortem CT Angiography in Children: A Study on a Porcine Model. Int J Legal Med (2018) 132(5):1391-403. doi: 10.1007/ s00414-018-1783-y

33. Mokrane F, Savall F, Dercle L, Crubezy E, Telmon N, Rousseau H, et al. Technical Note: A Preliminary Comparative Study Between Classical and Interventional Radiological Approaches for Multi-Phase Post-Mortem CT Angiography. Forensic Sci Int (2017) 271:23-32. doi: 10.1016/j.forsciint.2016.12.008

34. Ouchmich F, Angus V, Noblet V, Heitz F, Pessaux P. Liver Tissue Segmentation in Multiphase CT Scans Using Cascaded Convolutional Neural Networks. Int J Comput Assist Radiol Surg (2019) 14(8):1275-84. doi: 10.1007/s11548-019-01989-z

35. Putten J, Zinger S, Sommen F, With P, Prokop M, Hermans J. Quantitative CT Based Radiomics as Predictor of Resectability of Pancreatic Adenocarcinoma. Med Imaging 2018: Computer-Aided Diagnosis (2018) 10575O. doi: 10.1117/ 12.2291746

36. Ljosa V, Carpenter A. Introduction to the Quantitative Analysis of TwoDimensional Fluorescence Microscopy Images for Cell-Based Screening. PloS Comutational Biol (2009) 5(12):e1000603. doi: 10.1371/journal.pcbi.1000603

37. Farag A, Lu L, Roth H, Liu J, Turkbey E, Summers R. Automatic Pancreas Segmentation Using Coarse-to-Fine Superpixel Labeling. Deep Learn Convolutional Neural Networks Med Image Computing. Edition 1. (2017). p.279-302. doi: 10.1007/978-3-319-42999-1_16

38. Farag A, Lu L, Roth H, Liu J, Turkbey E, Summer R. A Bottom-Up Approach for Pancreas Segmentation Using Cascaded Superpixels and (Deep) Image Patch Labeling. IEEE Trans Image Process (2016) 26(1):386-99. doi: 10.1109/ TIP.2016.2624198

39. Salameh J, McInnes M, McGrath T, Salameh G, Schieda N. Diagnostic Accuracy of Dual-Energy CT for Evaluation of Renal Masses: Systematic Review and Meta-Analysis. Am J Roentgenol (2019) 212(4):W100-5. doi: 10.2214/AJR.18.20527

40. Sevcenco S, Spick C, Helbich T, Heinz G, Shariat S, Klingler H, et al. Malignancy Rates and Diagnostic Performance of the Bosniak Classification for the Diagnosis of Cystic Renal Lesions in Computed Tomography - a Systematic Review and Meta-Analysis. Eur Radiol (2017) 27(6):2239-47. doi: $10.1007 /$ s00330-016-4631-9 
41. Law R, Varayil J, Song L, Fidler J, Fletcher J, Barlow J, et al. Assessment of Multi-Modality Evaluations of Obscure Gastrointestinal Bleeding. World JWenal Gastroenterol (2017) 23(4):614-21. doi: 10.3748/wjg.v23.i4.614

42. Pepa R, Cerchione C, Pugliese N, Colicchio R, Salvatore P, Sirignano C, et al. Diagnostic-Driven Antifungal Approach in Neutropenic Patients at High Risk for Chronic Disseminated Candidiasis: Preliminary Observations on the Role of 1,3- $\beta$-D-Glucan Antigenemia and Multiphasic Contrast-Enhanced Computed Tomography. Supportive Care Cancer (2018) 36(6):1691-4. doi: 10.1007/s00520-018-4138-9

43. Georgiadou D, Sergentanis TN, Sakellariou S, Filippakis GM, Zagouri F, Vlachodimitropoulos D, et al. VEGF and Id-1 in Pancreatic Adenocarcinoma: Prognostic Significance and Impact on Angiogenesis. Eur J Surg Oncol (2014) 40(10):1331-7. doi: 10.1016/j.ejso.2014.01.004

44. Longo V, Brunetti O, Gnoni A, Cascinu S, Gasparini G, Lorusso V, et al. Angiogenesis in Pancreatic Ductal Adenocarcinoma: A Controversial Issue. Oncotarget (2016) 7(36):58649-58. doi: 10.18632/oncotarget.10765

45. Goel H, Mercurio A. VEGF Targets the Tumour Cell. Nat Rev Cancer (2013) 13:871-82. doi: $10.1038 / \mathrm{nrc} 3627$
Conflict of Interest: The authors declare that the research was conducted in the absence of any commercial or financial relationships that could be construed as a potential conflict of interest.

Publisher's Note: All claims expressed in this article are solely those of the authors and do not necessarily represent those of their affiliated organizations, or those of the publisher, the editors and the reviewers. Any product that may be evaluated in this article, or claim that may be made by its manufacturer, is not guaranteed or endorsed by the publisher.

Copyright (๐ 2021 Rahmanuddin, Korn, Cridebring, Borazanci, Brase, Boswell, Jamil, Cai, Sabir, Motarjem, Koay, Mitra, Goel, Ho, Chung and Von Hoff. This is an openaccess article distributed under the terms of the Creative Commons Attribution License (CC BY). The use, distribution or reproduction in other forums is permitted, provided the original author(s) and the copyright owner(s) are credited and that the original publication in this journal is cited, in accordance with accepted academic practice. No use, distribution or reproduction is permitted which does not comply with these terms. 